\title{
Treating a Dysregulated JAK/STAT Pathway in Cancer Cells
}

\author{
Jarod M. Schieler ${ }^{\mathrm{a}}$ and Jeffrey O. Henderson ${ }^{\mathrm{a}}$
}

\begin{abstract}
The JAK/STAT pathway is induced by the binding of a cytokine to its cognate receptor. The receptor's engagement with the cytokine recruits a JAK protein, which activates itself via auto/trans-phosphorylation. In turn, the activated JAKs recruit and phosphorylate STAT proteins. The phosphorylated STAT proteins form a dimer, translocates to the cell nucleus and acts as a transcription factor to induce gene expression. In this way, the JAK/STAT pathway can mediate a cell's response to extracellular signals. The proteins ultimately induced by the JAK/STAT pathway contribute to processes such as inflammatory response, differentiation, proliferation, and apoptosis. When the JAK/STAT pathway becomes dysregulated, proto-oncogenes and/or tumorsuppressor genes are often inappropriately expressed, commonly resulting in oncogenesis. This review discusses how SOCS, PIAS, and PTPS proteins modulate the JAK/STAT pathway ensuring that it remains cyclic and transient. The use of jakibins, STAT inhibitors, decoy oligonucleotides, RNA interference and genome editing to synthetically regulate a dysregulated JAK/STAT pathway in cancer cells are also considered.
\end{abstract}

Keywords: Cancer; CRISPR/Cas9; Cytokine; JAK/STAT; PIAS; PTPS; SOCS

\section{Introduction}

In America, nearly half of men and nearly one-third of women will develop cancer (American Cancer Society [ACS], 2015). There are over 200 different types of cancer and even more known and probable carcinogens (ACS, 2015). The first evidence of human cancer is written in ancient Egypt's hieratic script, named Edwin Smith Papyrus after the American archeologist who purchased the document, that dates to around 1600 B.C. (ACS, 2015). Cancer has a past and it is very much part of the present; however, millions of people around the world are working together to eradicate cancer so that it has no future. Because of soccer moms who partake in "cancer walks", researchers who spend countless hours peering through a microscope, and pharmaceutical companies that push new drugs through clinical trials, science is edging closer to understanding cancer.

There is no known single treatment plan for cancer, mostly because there are many types of cancer. However, all cancers have the same underlying characteristic. Oncogenesis, the formation and development of tumors, is the result of aberrant cell proliferation. All somatic cells undergo a natural process of cell division called mitosis. If mitosis is unregulated then uncontrolled cell growth can result in the formation of either a benign or malignant tumor. The latter type is life threatening if not treated early.

Abundant research in molecular biology has been conducted on tumorigenesis. Two classes of genes, protooncogenes and tumor-suppressor genes, play a key role in tumor formation. Proto-oncogenes are genes that provide signals that lead to cell division or regulate apoptosis. Gain-offunction mutations in proto-oncogenes that code for growth factors, growth factor receptors, signal-transduction proteins, transcription factors, pro/anti-apoptotic proteins, and DNA repair proteins generally give rise to dominantly active oncogenes (Schulz, 2007). Tumor-suppressor genes encode for proteins that inhibit cell growth and tumor development (ACS, 2015). However, mutated tumor suppressor genes that code for cell cycle control proteins such as cyclin D1 may contribute to tumorigensis (Schulz, 2007). Genes are transcribed and translated into proteins that may take part in important biochemical pathways. Pathways are chemical chain reactions that participate in maintaining natural homeostasis inside cells. Cell signal transducing pathways are pathways that are prompted by the binding of a messenger to an external receptor. A signal-transducing pathway that is relevant to oncogenesis is the JAK/STAT pathway.

Janus Kinase/Signal Transducer and Activator of Transcription (JAK/STAT) pathway is appropriately named after the Roman god of passageways. The JAK/STAT pathway is pleiotropic because it is responsible for transducing a multitude of signals that lead to inflammatory responses, differentiation, cell migration, apoptosis, and proliferation (Rawlings et al., 2004). The latter three responses, if inappropriately activated, are most commonly seen in cancer. Inappropriate expression of genes that control apoptosis and proliferation is fundamental in cancer. Once cancer forms, it becomes more difficult to quarantine and treat once metastasis occurs. The JAK/STAT pathway is kept transient and cyclic under normal conditions. However, if any components of the JAK/STAT pathway behave atypically, inappropriate signaling may result. The JAK/STAT pathway brings about cellular responses by directly affecting transcription. If the pathway itself behaves aberrantly then it will inappropriately affect transcription. The proteins that arise from inappropriate levels of transcription may be atypical in function or expression level, potentially affecting cell homeostasis and leading to diseases such as cancer (Costa-Pereira et al., 2011).

\section{Components of the JAK/STAT pathway}

\section{Cytokine Receptor}

The cytokine receptor is the first component of the JAK/STAT pathway. Type I cytokine receptors are more commonly linked to cancer than other cytokine receptor families: Type II, chemokine, tumor necrosis factor, TGF-beta, and immunoglobulin receptors (Murray et al., 2007). 
Cytokines include small peptides, proteins, and glycoproteins whose primary function is to help carry out paracrine communication by binding their cognate receptor (Jatiani et al., 2010). A common cytokine that binds Type I cytokine receptors is interleukin 6 (IL-6). Interleukins typically serve as communication links between leukocytes; however, IL-6 is a multifunctional cytokine that is involved in the modulation of growth and differentiation in tumors of multiple cancers including myeloma, non-small cell lung carcinoma, colorectal, renal cell carcinoma, prostate, breast and ovarian (Guo et al., 2012; Wormald and Hilton, 2004). Cytokine I receptors, like IL-6R, consist of two or more subunits, notably an extracellular cytokine binding domain averaging 210 amino acids in length with the hallmark W-S-XW-S motif of cytokine I receptors, and an intracellular signaltransducing domain that can activate JAK proteins once a cytokine (e.g. IL6) binds to it (Jatiani et al., 2010).

\section{JAK}

The second component in the transduction of cytokinemediated signals through the JAK/STAT pathway is JAK. The four types of Janus Kinases are JAK1, JAK2, JAK3, and TYK 2.

All of the families share seven conserved homology domains (Jatiani et al., 2010). JAKs are named after the twofaced god, Janus, because the C-terminus has two complementary domains: $\underline{J} A K$ homology domain number $\underline{1}$ (JH1), the active catalytic tyrosine-kinase domain, and $\mathrm{JH}_{2}$, which is an inactive non-catalytic regulatory pseudokinase domain that is thought to negatively regulate $\mathrm{JH} 1$ (Yamaoka $e t$ al., 2004). JH5, JH6, and JH7 represent the FERM (four-pointone, ezrin, radixin, and moesin) domain of the protein (Yamaoka et al., 2004). The FERM domain promotes the protein's ability to be recruited and bound to cytokine receptors once the cognate cytokine engages with the cytokine receptor (Jatiani et al., 2010).

Once JAK proteins are bound to the intracellular signaltransducing domain the proteins auto/trans-phosphorylate themselves and the tyrosine residues on the intracellular cytoplasmic domain of the cytokine receptor. Once activated, the STAT proteins bind to the phosphorylated tyrosine residues (Yamaoka et al., 2004). The JH3 and JH4 domains of JAK constitute the Src $\mathrm{H} 2$ binding domain (SH2) that allows binding to molecules containing an $\mathrm{SH} 2$ domain. JAKs are important because they mediate the phosphorylation of cytokine receptors and create docking sites on their intracellular domains for binding of $\mathrm{SH}$ 2-containing proteins like STATs (Jatiani et al., 2010).

\section{STAT}

STAT activation is the next step in the JAK/STAT pathway. STAT proteins reside latently in the cytoplasm until they are recruited by an activated JAK that is bound to the intracellular cytoplasmic domain of a cytokine receptor (Rawlings et al., 2004). There are two predominant mechanisms for how STAT proteins can become activated. STAT proteins can be phosphorylated either by a JAKcytokine receptor intracellular domain complex or by the phosphorylated tyrosine domains on the cytokine receptor intracellular domain (Jatiani et al., 2010). For example, if a STAT molecule associates with phosphorylated Y701 in the
SH2 domain of a JAK protein, the STAT protein itself becomes activated (Liu et al., 1998).

STAT (signal transducer and activator of transcription) has seven mammalian family members: STAT1, STAT2, STAT3, STAT4, STAT5A, STAT5B, and STAT6. STATs have six domains: N-terminal domain, coiled-coil domain, a unique DNA binding domain that allows each STAT to bind to and regulate a distinct subset of genes, linker domain, $\mathrm{SH} 2$ domain, and a tyrosine/serine transcriptional activation domain (Bowman et al., 2000). Once two STAT molecules become phosphorylated they form a homo/heterodimer and are shuttled to the nucleus by an importin $\alpha-5$ dependent mechanism (Meyer et al., 2004). Once inside the nucleus, the STAT dimer binds to the GAS-like (gamma interferon activated sites) promoter element, which is an $8-10 \mathrm{bp}$ inverted repeat with the consensus sequence 5'-TT(N5)AA-3' (Ehret et al., 2001). The STAT-DNA interaction activates transcription of downstream interferon-stimulated genes known to code for proteins that participate in cell growth and proliferation, such as c-myc transcription factor. The c-myc gene, located on chromosome 8 , is estimated to modulate the expression of approximately $15 \%$ of all genes in the human body (Gearhart et al., 2007).

\section{Aberrant JAK/STAT Pathway}

Cytokine receptors, JAKs, and STATs are all integral constituents of the JAK/STAT pathway. Somatic gain-offunction alterations in JAK-STAT signaling can lead to uncontrolled cell proliferation that drives cancer growth and myeloproliferative diseases (Kovacic et al., 2006; Witte et al., 2014). Cancer may arise if genes, like proto-oncogenes or tumor-suppressor genes, are altered by spontaneous mutation or carcinogen induced mutation. Mutations can cause an important regulatory protein to not be encoded for or cause a mutated protein that has aberrant functionality. This section discusses pertinent genetic mutations, and focuses on the molecular level of alterations in JAK/STAT signaling that can lead to cancer.

\section{Dysregulation of Cytokine Signaling}

If the extracellular compartment contains a large concentration of cytokines then there will be a higher probability that they will find their cognate receptor on extracellular membranes. If there is more cytokine engagement with a receptor then the JAK/STAT pathway may cease to be transient and could eventually lead to a greater level of transcription. For example, increased levels of IL-6, one of the most ubiquitously deregulated cytokines in cancer, have been observed in nearly every tumor studied (Hong et al., 2007). Cytokine receptor signaling is largely regulated by SOCS (discussed below) and nuclear hormone receptors (Leung et al., 2004). Nuclear hormone receptors, which are a class of ligandactivated proteins, may regulate cytokine production and modulate the expression and signaling of cytokine action (Leung et al., 2004).

\section{Mutated JAKs}

Genetic mutations of JAK1, JAK2, and JAK3 are commonly seen in cancers. For instance, a somatic gain-offunction mutation, $\mathrm{V} 617 \mathrm{~F}$, in the pseudokinase $\mathrm{JH} 2$ domain of JAK2, is seen in patients suffering from myeloproliferative disorders such as polycythemia vera (a neoplasm in which the bone marrow makes too many red blood cells) (Baxter et al., 
2005). Recall that JH2, a pseudokinase domain, is thought to inhibit the role of the $\mathrm{JH} 1$ kinase domain. If the $\mathrm{JH} 2$ domain is mutated then its ability to negatively regulate the phosphorylation of $\mathrm{JH} 1$ is inhibited. Without the important negative regulatory function of the $\mathrm{JH} 2$ domain, the active catalytic tyrosinase-kinase domain will become unregulated and promiscuously autophosphorylate tyrosine residues and transphosphorylate the intracellular cytoplasmic domain of cytokine receptors. Therefore, an inappropriately activated JH1 domain is more likely to participate in unrestrained recruiting of STAT molecules.

Normal JAK functions may be perturbed on the molecular level as well. For example, JAK may be atypically activated if there is a genetic mutation in a DNA segment that no longer encodes for an effective regulatory protein like PTP (discussed below) that assists in the dephosphorylation of JAK.

\section{Constitutively Activated STAT}

Normally, STAT activity is kept transient by proteins that act as down-regulators in the cell, but STAT activity in cancer cells is largely unregulated when genes that code for proteins such as SOCS and PIAS (discussed below) are mutated. Theoretically, if a mutated STAT binds to a DNA segment that it normally would not, then the abnormal expression of genes (e.g. proto-oncogenes) may result. At the molecular level, constitutively activated STAT proteins are common hallmarks seen in many cancers (Bromberg et al., 1996; Kovacic et al., 2006). If a STAT molecule is constitutively activated they, as an unregulated transcription factor, constantly induce the transcription for genes that lay downstream of the GAS element it binds to (Matsumoto et al., 1997). Constitutive activation of STAT proteins leads to induction of genes involved in controlling cell proliferation and survival (Bowman et al., 2000). Some interferon stimulated genes that an activated STAT protein can induce transcription of are myc and bcl-xl. Myc, as described above, encodes for an important transcription factor (also called myc) that is responsible for inducing the transcription of genes that are involved with cell growth and proliferation. Bcl-xl proteins are important to cancer cells because they inhibit an important intrinsic apoptotic pathway by regulating mitochondrial outer membrane permeability. If bcl-xl proteins are present, they will inhibit the release of cytochrome $\mathrm{C}$ into the cytsol through an interaction with the outer mitochondrial membrane transition pores (Zamzami et al., 1998). Cytochrome C is an important intermediate in the process of apoptosis. Therefore, if the release is inhibited by bcl-xl, then the cancer cell is more likely to evade the apoptotic checkpoint induced by DNA damage. STATs bind to slightly different elements and can therefore induce the transcription of different genes (Zamzami et al., 1998). For example, STAT5 binds to DNA segments that encode for proteins supporting cell survival and proliferation (Matsumoto et al., 1997). Studies have suggested that STAT3 alone is sufficient to induce neoplastic transformation (Sen $e t$ al., 2002). STAT3 is also a well-documented oncogene known to activate the transcription of genes necessary for the progression of the cell cycle (Bromberg et al., 2006). For example, STAT3-DNA binding increases the transcription of CCND1 gene that codes for cyclin D1 (Leslie et al., 2006). Cyclin D1 forms a complex with a cyclin-dependent kinase that is necessary for the progression of mitotic $\mathrm{G}$ to $\mathrm{S}$-phase (Bromberg et al., 2006). Activated STAT3 correlates with elevated cyclin D1 protein in primary breast tumors and breast cancer-derived cell lines (Leslie et al., 2006). Furthermore, the presence of most tumors correlates significantly with expression levels of several STAT3-downstream proangiogenic genes. Proangiogenic genes encode proteins that promote angiogenesis, which is the process through which blood vessels are formed. The formation of blood vessels is important to cancer because a growing tumor needs an increasing supply of oxygen and nutrients to sustain it and to eventually metastasize throughout the body (Wei et al., 2003; Yang et al., 2010).

Interestingly, not all STAT proteins activate a gene that leads to cell proliferation and survival. In fact, STAT1 has been shown to have the opposite effect of STAT3 by promoting apoptosis and inhibiting angiogenesis (Huang et al., 2002). However, STAT1 activity is often inhibited as cells become unresponsive to the growth-inhibitory effects of interferons (Shulz, 2007).

\section{Somatic Regulation of the JAK/STAT Pathway}

\section{SOCS}

The SOCS (ㅁuppressors of cytokine signaling) family has eight members: SOCS1-7 and CIS. All have a SH2 domain (that can bind to molecules with SH2-docking domains) and a SOCS box (40 amino acid module) at the C-terminus (InagakiOhara et al., 2013). Kyoko Inagaki-Ohara's review (2013) suggests that SOCS can inhibit cytokine signaling in four distinct ways. First, SOCS can block STATs recruitment to the cytokine receptor by competing for, and thereby masking, $\mathrm{SH} 2$ binding sites of the intracellular domain of the cytokine receptor. For example, Matsumoto and colleagues (1997) discovered that a member of the SOCS family, CIS (cytokineinducible $\underline{\mathrm{S}} \mathrm{H} 2$-containing protein), inhibits the interaction of cytokine receptor and STAT5. They found that the CIS gene promoter region contains MGF boxes to which STAT5 binds, inducing the expression of the CIS protein (negative feedback loop). CIS protein competes with STAT5 for binding on the activated cytoplasmic tail of type I or type II cytokine receptors. CIS can also bind directly to STAT5. Both scenarios bring about the same result - a prevention of STAT5 activation via phosphorylation. A second way that SOCS can inhibit cytokine signaling is by targeting STATs for ubiquitin mediated proteosomal degradation. Specifically, the SOCS box has E3 ubiquitin ligase activity allowing it to participate in the targeted degradation of STATs (Matsumoto et al., 1997). Thirdly, SOCS can bind to the SH2-docking domain on STAT and inhibit its ability to transphosphorylate. And finally, SOCS can also target JAKs for proteasomal degradation.

\section{PIAS}

Five different types of PIAS (protein inhibitors of activated stats) have been identified: PIAS1, PIAS3, PIASy, PIASx, and PIASx- $\beta$, all of which have a zinc binding motif. Like SOCS, PIAS are thought to modulate the JAK/STAT pathway in a variety of different ways. One way is that PIAS proteins inhibit STAT from binding to DNA (Shuai et al., 2006). PIAS bind directly to STATs, blocking protein-DNA interactions using two different mechanisms: the binding of a PIAS protein to a STAT dimer may mask the DNA binding domain of STAT, or 
PIAS proteins may bind to STATs and prevent their dimerization (Liu et al., 1998). For example, PIAS1 and PIAS3 inhibit STAT-mediated gene activation by blocking DNA binding (Liu et al., 1998).

Another way that PIAS proteins modulate the JAK/STAT pathway is through chromatin remodeling. Chromatin remodeling involves the balance between at least two classes of enzymes histone acetyltransferases (HAT) and histone deacetyltransferases (HDAC). Inside the cell nucleus genes are normally packaged into tight structures called chromosomes. Chromosomes contain many subunits called chromatin an aggregate of DNA and histone proteins. The fundamental repeating submit of chromatin is the nucleosome consisting of a core particle composed of an octamer of histone proteins around which a length of DNA containing about 145 nucleotides is wound. The degree of how tightly the DNA is wrapped around the histones is determined by HAT and HDAC. HAT enzymes loosen the DNA-histone interaction by acetylating (and thereby neutralizing the positive charge that attracts it to the negatively charged DNA) the histone. This interaction forms an open chromatin structure that can be more easily accessed by transcriptional machinery such as RNA polymerase and transcription factors. Conversely, HDAC is a family of enzymes that remove the acetyl groups from histones (leaving the histone with a positive charge that is attracted to the negatively charged backbone of DNA) allowing for tight DNA coiling. The DNA wrapping, specifically 1.65 times, is sufficient enough to repress transcription (Weaver, 2012). For example, PIASy, PIASx, and PIASx- $\beta$ recruit histone deacetylases to repress the transcriptional activity of STAT proteins (Shuai et al., 2006).

Finally, PIAS proteins are also thought to regulate STATS by promoting SUMOylation, the addition of small ubiquitinlike modifier proteins. In many cases, SUMO modification of STAT proteins correlates with inhibition of STAT dependent transcription (Gill et al., 2005).

\section{PTPs}

Some of the more common members of the PTP (Proteins tyrosine phosphatases) family are SHP1, SHP2, TC45, and CD45 which all contain an SH2 domain that allows them to bind to STATs and JAKs. Specifically TC45 is responsible for the dephosphorylation of STAT1 in the nucleus (Hoeve et al., 2002). The JAK/STAT pathway is driven by kinases. Cytokine receptors, JAKs, and STATs are all important components that carry out their function because of their ability to be phosphorylated and/or phosphorylate the next member in the pathway. PTPS are important enzymes essential for a cell's ability to negatively regulate the JAK/STAT pathway preventing uncontrolled transcription.

\section{Synthetic Regulators of Unregulated Jak/Stat Pathway}

When the somatic regulators in the body are ineffective or absent, uncontrolled transcription can result from an inappropriately overactive JAK/STAT pathway. Carcinogens can mutate the genes that code for tumor-suppressor genes, proto-oncogenes, and many of the vital somatic regulators of the STAT pathway such as SOCS, PIAS, and PTPS. Since oncogenesis is caused by aberrant cell proliferation, much research is being done to learn how synthetic modulators can be implemented into the proteome of cancer cells to carry out the intended function of the overwhelmed or ineffective somatic regulators.

\section{Jakinibs}

JAK inhibitors are a family of kinase inhibiters collectively called Jakinibs. There are currently 19 FDA-approved Jakinibs. Clinically tested Jakinibs can inhibit more than one of the three other JAKs: JAK1, JAK2, and JAK3. For example, the first JAK inhibitor to be discovered, tofactinib, primarily inhibits JAK3, but has also been shown to inhibit JAK2 and JAK1 (Ghoreschi et al., 2011). Because certain cancers have the ability to develop resistance to drugs that target a specific JAK the emergence of nonspecific kinase inhibitors, such as tofactinib, have become clinically useful (Kontzias et al., 2012). However, because different types of JAKs can interact with the same types of STAT molecules, the inhibition of one specific JAK/STAT interaction does not necessarily eradicate the cancer. The first FDA-approved Jakinib was ruxoltinib, which inhibits both JAK1 and JAK2. Currently ruxoltinib is being used to treat myeloproliferative diseases such as lymphomas, pancreatic cancer, and polycythemia vera. Unfortunately, there are no Jakinibs that selectively target Tyk2 being tested in clinical trials (Kontzias et al., 2012). This is a significant limitation because there are many cancers that can still be enhanced by Tyk 2 expression. For example, results from a study conducted by Ide and coworkers (2008) using small interfering RNAs targeting Tyk2 suggests that Tyk2 signaling is involved in the metastasis of prostate cancer cells.

\section{STAT Inhibitors}

Constitutively activated STAT3 is a hallmark of cancer in the breast, pancreas, prostate, and lungs (Turkson et al., 2005). As a transcription factor that regulates the expression of genes that control the cell cycle, constitutively activated STATs play an important role in tumorigenesis and are novel targets for oncogenic therapeutic intervention. Blocking STAT3 or STAT5 function alone has been demonstrated to be sufficient to inhibit tumor cell growth and induce apoptosis (Buettner et al., 2002; Xiong et al., 2014). Inhibitors that target STAT3 include peptidomimetics, metal compounds, small molecule inhibitors, and oligonucleotides (Furqan et al., 2013).

Small molecules comprise the largest class of STAT inhibitors. Small molecule inhibitors interact with the SH2 domain of STATs and hamper STAT dimerization and transcriptional activity (Furqan et al., 2013). Inhibitors, such as $\mathrm{XZH}-5$, bind to the $\mathrm{SH} 2$ domain of STAT molecules, thereby preventing the phosphorylation of the Tyr705 region (Furqan et al., 2013). The phosphorylation by an activated JAK or intracellular cytoplasmic domain of an activated cytokine receptor is what activates the STAT itself. This inhibition has been shown to lead to apoptosis in breast cancer, pancreatic cancer, hepatoceullular carcinoma, and rhabdomyosarcoma (Liu et al., 2012). Another STAT inhibitor is Stattic, which specifically prohibits STAT3 dimerization. Recall that dimerization is an essential step before activated STAT molecules can be translocated to the nucleus by an importin $\alpha-5$ dependent mechanism. (Meyer et al., 2004).

Peptidomimetics also prevent STAT dimerization. Peptidomimetics are compounds that are designed to mimic a peptide. The peptidomimetic sequence, PY*LKTK, where Y* represents phosphotyrosine, is able to bind to the $\mathrm{SH} 2$ domain 
essential for STAT binding. Without being able to form a hetero/homo dimer the activated STAT molecules transcriptional activity, on proto-oncogenes such as src, is inhibited (Turkson et al., 2001). While STAT4 is a lesserknown oncogene, targeted disruption of the STAT4 gene in mice demonstrated that STAT4 is required for induced proliferation of T cells (which may lead to T-cell lymphoma). Unfortunately, no promising STAT4 dimerization inhibitor has yet been developed (Furqan et al., 2013).

\section{Conclusion and Prospectus}

All cancer, no matter what type, has the same foundational feature: the uncontrolled growth of cells. It's ironic that the very process that is necessary to sustain a life also has the potential to develop into a cancer that ends a life.

In recent decades, technological advances in gene knockdown through mechanisms such as RNA interference have allotted for a proliferation of discoveries in cancer research. The research surrounding the JAK/STAT pathway is a small part of the puzzle; however, it has contributed much to our understanding of how aberrant pathways can cause cancer by bypassing apoptosis and cell cycle checkpoints. The studies described in this review suggest that components of the JAK/STAT pathway may serve as important therapeutic targets for cancer therapy. Because mutated components are expressed from altered genes it seems plausible that research focused on the interactions between aberrant molecules, such as inappropriately activated JAKs or STATs, are ends for attacking the flames of the fire, rather than the base. Although interesting research on the modulation of an unregulated JAK/STAT pathway is being done, perhaps, a more efficient way to treat genetic diseases involves the use of a nucleic acidbased approach. Since the Human Genome Project (2003) was completed, researchers have identified specific DNA sequences that lead to many genetic diseases such as cancer, sickle cell anemia, and cystic fibrosis.

Decoy oligonucleotides are being used to bind to specific STAT recognition DNA sequences $\left(5^{\prime}-\mathrm{TT}\left(\mathrm{N}_{5}\right) \mathrm{AA}-3^{\prime}\right)$ to attenuate binding of the endogenous STAT dimer transcription factor (Ehret et. al., 2001). A double-stranded short oligonucleotide that also selectively binds to the GAS promoter element can be synthesized. Decoy oligonucleotides that mimic transcription factors E2F-1, CREB and NFאB have been shown to result in strong suppression of cell proliferation and induction of apoptosis because of their ability to bind sequences that the endogenous transcription factors bind (Fang et al., 2011; Furqan et al., 2013). If two molecules with similar affinities toward the GAS promoter element are competing for binding, the overall binding interaction of them both should be less than if there was no competition at all. However, decoy oligonucleotides can also be synthesized to bind not to the DNA sequence of interest, but rather to the STAT molecule itself. Decoys can be made to bind to dimerized phosphorylated STAT3 or STAT5, thereby preventing STAT/DNA interaction (Barton et al., 2004).

RNA interference is a sequence specific posttranscriptional nucleic-acid based approach that involves dsRNA, Dicer, and a RISC complex. Dicer resembles RNase II and is able to degrade dsRNA molecules into roughly 22 nucleotide fragments called siRNA. The siRNAs are then integrated into RISC (ㅁNA-induced silencing complex) where they undergo complementary base pairing to a target mRNA so the RISC complex can cleave it (Weaver, 2012). The cleaving of the mRNA leaves it unable to serve as a template suitable enough for a ribosome to translate. The aforementioned process makes siRNA naturally, however synthetic RNAs can also be implemented into the silencing process. Scientists are able to silence specific gene sequences by transfecting a specific siRNA sequence into the cell. RNA interference is beginning to prove efficacious in silencing an inappropriately active gene that causes a disease. For example, interference of STAT3 signaling using siRNA significantly suppressed growth of tumors, induced apoptosis, and down- regulated important proteins necessary for cancer cell survival such as Bcl-2 (an important anti-apoptoic protein) and cyclin D1 (Gao et al., 2006). Recall that cyclin D1 is important in the progression of mitotic $\mathrm{G}$ to $\mathrm{S}$-phase. If cylcin D1 expression is downregulated, the progress of mitosis is slowed, and cell growth is consequently slowed as well. The technology of RNA interference seems promising; however, there is appropriate concern that genes with similar sequences can be silenced as well. This type of silencing, classified as an off-target effect, is an obstacle that could prove detrimental if the expression of a vital protein is also silenced as well.

While decoy oligonucleotides are involved with masking binding sites on DNA sequences and RNA interference involves cleaving target mRNA, both of these techniques lack the ability to rewrite the genetic code.

In the past decade, 'genome editing' has emerged as a promising technology to evolve the field of personalized medicine. Genome editing is based on the use of engineered nucleases composed of sequence-specific DNA-binding domains fused to a nonspecific DNA cleavage module (Gaj et al., 2013). Unlike conventional methods, which either temporarily address disease symptoms or randomly integrate therapeutic factors in the genome, ZFNs (Zinc-finger nucleases), TALENs (Transcription activator-like effector nucleases), and the three CRISPR/Cas systems (luster regulatory interspace short palindromic repeats) are capable of correcting the underlying cause of the disease, therefore permanently eliminating the symptoms with precise genome modifications (Gaj et al., 2013).

Bacteria have a natural process of acquiring immunity against viruses. The bacterium is able to detect the viral DNA, and integrate small pieces of it within the CRISPR locus on a chromosome. The CRISPR locus can be transcribed into short crRNA (CRISPR targeting $\underline{\text { RNA) }}$ ) that anneal to tracrRNAs (transactivating crRNAs) forming a duplex structure that recruits the endonuclease Cas9 (Esvelt et al., 2013). Within the complex, a spacer on the $5^{\prime}$ end of crRNA base-pairs with a complementary sequence on the targeted viral DNA with a protospacer that lies upstream of a highly conserved motif PAM (protospacer adjacent motif) on the crRNA (Esvelt et al., 2013). The binding can be modulated by changing the sequence of the spacer on the crRNA. This is an important advantage over TALEN and ZFN; in this way the CRISPR/Cas9 system can quickly and cheaply be retargeted to cleave virtually any DNA sequence by simply redesigning the crRNA that is injected into the cell (Gaj et al., 2013). TALENs and ZFNs are modular DNA-binding proteins that are site-specific nucleases. These technologies require constructing a protein for each specific target DNA sequence to be edited. The CRISPR/Cas9 involves a RNA-guided DNA endonuclease that can be used for any DNA gene sequence. Instead of designing a new 
protein for each sequence, the CRISPR/Cas9 system can be easily adapted by redesigning the spacer of the crRNA inside it. Thus, TALENs and ZFNs can take relatively longer time, cost more, and are associated with a higher degree of difficulty than the CRISPR/Cas9 system (Gaj et al., 2013). Once the crRNA/target DNA hybrid is formed, the Cas9 endonuclease acts as a "molecular scalpel" and makes a double-stranded DNA break exactly three nucleotides upstream of the PAM. (Esvelt et al., 2013). This precision is incredibly important when a cut one nucleotide more or less than three can cut into a different codon shifting the reading frame. Once the double stranded break has been completed, the cell naturally attempts to repair the DNA by either NHEJ (non homologous end joining) or HR (homology recombination). NHEJ involves the ligation of the cleaved ends from the target DNA, usually with the inclusion of an insertion or deletion that will disrupt the reading frame of the desired gene, which can lead to the formation of a premature stop codon (UAG, UGA, UAA) and often results in gene silencing ( $\operatorname{Ran}$ et al., 2013). HR is the other repair pathway, and is associated with gene editing rather than silencing (Ran et al., 2013). The advantage to this pathway is that a donor DNA molecule, that has arms that match the flanking sequences on the cleaved target DNA, can be inserted in order to change the gene of interest on the target DNA (Ran et al., 2013).

The ability to edit a gene that is known to cause a genetic disease is an incredibly fascinating milestone in science. It seems promising that technology such as CRISPR /Cas9 will revolutionize personalized medicine. Imagine a cancer patient in which the exact genetic mutation that causes their disease is known. With technologies such as the CRISPR/Cas9 system the physician doesn't have to use the scalpel and invasive surgeries to attack the symptoms that arise from the genetic mutation, instead the physician can attack the base of the fire by utilizing a personalized pharmaceutical drug that uses the tiny molecular scalpel that bacteria have used for millions of years! The CRISPR/Cas9 system allows for the mutated gene to be cut out and with the use of HR repair, a perfect nonmutated sequence can be inserted directly into the genome so the correct protein can be coded for and the body can heal itself. The system could be personalized by altering the sequence within the spacer of the crRNA that can complementary base pair with the known mutated sequence within the patient's genome. Mutated genes such as genes that encode for aberrant constituents in the JAK/STAT pathway or tumor-suppressor genes and proto-oncogenes that can disrupt the cell's ability to control its own growth can theoretically be rectified with this game-changing technology.

\section{REFERENCES}

American Cancer Society. [Internet]. Accessed 2015 Jun 29. Available from http://www.cancer.org

Barton, B.E., Murphy, T.F., Shu, P., Huang, H.F., Meyenhofer, M., \& Barton, A. (2004). Novel single-stranded oligonucleotides that inhibit signal transducer and activator of transcription 3 induce apoptosis in vitro and in vivo in prostate cancer cell lines. Molecular Cancer Therapy. 10, 1183-91.

Baxter, E.J., Scott, L.M., Campbell, P.J., East, C., Fouroclas, N., Swanton, S., Vassiliou, G.S., Bench, A.J., Boyd, E.M., Curtin, N., Scott, M.A., Erber, W.N., \& Green, A.R. (2005).
Acquired mutation of the tyrosine kinase JAK2 in human myeloproliferative disorders. Lancet. 365, 1054-61.

Bowman T., Garcia R., Turkson J., \& Jove R. (2000). STATs in oncogenesis. Oncogene.19, 2474-2488.

Bromberg J.F., Wrzeszczynska M.H., Devgan G., Zhao Y., Pestell R.G., Albanese C., \& Darnell J.E (1996). STAT3 as an oncogene. Cell. 98, 295- 303.

Buettner, R., Mora, L.B., \& Jove, R. (2002). Activated STAT signaling in human tumors provides novel molecular targets for therapeutic intervention. Clinical Cancer Research. 4, 945-54.

Costa-Pereira, A. P., Bonito, N.A., \& Seckl, M.J. (2011). Dysregulation of janus kinases and signal transducers and activators of transcription in cancer. American Journal of Cancer Research. 1(6), 806-816.

Ehret, G.B., Reichenback, P., Schindler, U., Horvath, C.M., Fritz, S., Nabholz, M., \& Bucher, P. (2001). DNA binding specificity of different STAT proteins. Comparison of in vitro specificity with natural target sites. Journal of Biological Chemistry. 9, 6675-88.

Esvelt, K.M. Mali, P., Braff, J.L., Moosburner, M., Yaung, S.J., Church, G.M. (2013). Orthogonal Cas9 proteins for RNAguided gene regulation and editing. Nature Methods. 10, 1116-21.

Fang, Y., Sun, H., Zhai, J., Zhang, Y., Yi, S., Hao, G., \& Wang, T. (2011). Antitumor activity of NF-kB decoy oligodeoxynucleotides in a prostate cancer cell line. Asian Pacific Journal of Cancer Prevention. 10, 2721-6.

Furqan, M. Mukhi, N., Lee, B., \& Liu, D. (2013). Dysregulation of JAK-STAT pathway in hematological malignancies and inhibitors for clinical application. Biomarker Research. 1:5.

Gaj, T., Gersbach, C.A., \& Barbas III, C.F. (2013) ZFN, TALEN, and CRISPR/Cas-based methods for genome engineering. Trends in Biotechnology. 31(7):397-405.

Gao, L.F., Wen, L.J., Yu, H., Zhang, L., Meng, Y., Shao, Y.T., $\mathrm{Xu}$, D.Q., \& Zhao, X.J. (2006). Knockdown of Stat3 expression using RNAi inhibits growth of laryngeal tumors in vivo. Acta Pharmacologica Sinica. 3, 347-52.

Gearhart, J. Pashos, E., \& Prasad, M.K. (2007). Pluripotency redux--advances in stem-cell research. New England Journal of Medicine. 357, 1469-1472.

Ghoreschi, K., Jesson M.I., Li, X., Lee, J.L., Ghosh, S., Alsup, J.W., Warner, J.D., Tanaka, M., Steward-Tharp S.M., Gadina, M., \& Thomas, C.J. (2011). Modulation of Innate and Adaptive Immune Responses by Tofacitinib. Journal of Immunology. 186, 4234-43.

Gill, G. (2005). Something about SUMO inhibits transcription. Current Opinion in Genetics \& Development. 15, 536-41.

Guo, Y., Xu, F., Lu, T., Duan, Z., \& Zhang, Z. (2012). Interleukin-6 signaling pathway in targeted therapy for cancer. Cancer Treatment Reviews. 7, 904-10.

Hoeve, J.T., Maria, D.J.I.S., Yubin, F., Wei, Z., Michel, T., Michael, D., \& Ke, S. (2002). Identification of a Nuclear Stat1 Protein Tyrosine Phosphatase. Molecular and Cellular Biology. 16, 5562-5668.

Hong, D.S., Angelo, L.S., \& Kurzrock, R. (2007). Interleukin6 and its receptor in cancer: implications for Translational Therapeutics. Cancer. 110, 1911-1928.

Huang, S., Bucana, C., Van Arshall, M., \& Fidler, I. (2002). Stat1 negatively regulates angiogenesis, tumorigenicity and metastasis of tumor cells. Oncogene. 21, 2504-2512. 
Ide, H., Nakagawa, T., Terado, Y., Kamiyama, Y., Muto, S., \& Horie, S. (2008). Tyk2 expression and its signaling enhances the invasiveness of prostate cancer cells. Biochemical and Biophysical Research Communications. 369(2):292-6. Epub 2007 Sep 5.

Inagaki-Ohara, K., Kondo, T., Ito, M., \& Yoshimura, A. (2013). SOCS, inflammation, and cancer. JAKSTAT. 3, e24053.

Jatiani, S.S., Baker, S.J., Silverman, L.R., \& Reddy, E.P. (2010). JAK/STAT pathways in cytokine signaling and myeloproliferative disorders: approaches for targeted therapies. Genes \& Cancer. 10, 979-93.

Kontzias, A., Kotlyar, A., Laurence, A., Changelian, P., O'Shea, J.J. (2012). Jakinibs: A new class of kinase inhibitors in cancer and autoimmune disease. Current Opinions in Pharmacology. 4, 464-70.

Kovacic, B., Stoiber, D., Moriggl, R., Weisz, E., Ott, R.G., Kreibich, R., Levy, D.E., Beug, H., \& Freissmuth, M. (2006). STAT1 acts as a tumor promoter for leukemia development. Cancer Cell. 10, 77-87.

Leslie, K. Lang, C. Devgan G., Azare, J., Berishaj, M., Gerald, W., Kim, Y.B., Paz, K., Darnell, J.E. Albanese, C., Sakamaki, T., \& Pestell, R., Bromberg, J. (2006). Cyclin D1 is transcriptionally regulated by and required for transformation by activated signal transducer and activator of transcription 3. Cancer Research. 66, 2544-52.

Leung, K.C. (2004). Regulation of cytokine receptor signaling by nuclear hormone receptors: a new paradigm for receptor interaction. DNA and Cell Biology. 23, 463-74.

Liu, A., Liu, Y., Jin, Z., Hu, Q., Lin, L., Jou, D., Yang, J., Xu, Z., \& Wang, H. (2012) XZH-5 inhibits STAT3 phosphorylation and enhances the cytotoxicity of chemotherapeutic drugs in human breast and pancreatic cancer cells. PLoS One. 7, e46624.

Liu, B., Liao, J., Rao, X., Kushner, S.A., Chung, C.D., Chang, D.D. \& Shuai, K. (1998) Inhibition of Stat1- mediated gene activation by PIAS1. Proceedings of the National Academy of Sciences U.S.A. 95, 10626-31.

Schulz, WA. (2007). Molecular Biology of Human Cancers: An Advanced Student's Textbook. New York: W.H. Freeman.

Matsumoto, A., Masuhara, M., Mitsui, K., Yokouchi, M., Ohtsubo, M., Misawa, H., Miyajima, A. \& Yoshimura, A. (1997). CIS, a cytokine inducible SH2 protein, is a target of the JAK-STAT5 path- way and modulates STAT5 activation. Blood. 89, 3148-3154.
Meyer, T., \& Vinkemeier, U. (2004). Nucleocytoplasmic shuttling of STAT transcription factors. European Journal of Biochemistry. 271, 4606-12.

Murray, P. (2007). The JAK-STAT signaling pathway: input and output integration. Immunology. 5, 2623-2629

Ran, F.A., Hsu, P.D., Wright, J., Agarwala, V., Scott, D.A., Zhang, F. Genome engineering using the CRISPR-Cas9 system. Nature Protocols. 8(11):2281-2308.

Rawlings, J.S., Rosler, K.M., \& Harrison, D.A. (2004). The JAK/STAT signaling pathway. Journal of Cell Science. 117, 1281-1283.

Sen, M. \& Grandis, J.R. (2012). Nucleic acid-based approaches to STAT inhibition. JAKSTAT. 4, 285-91.

Shuai, K. (2006). Regulation of cytokine signaling path-ways by PIAS proteins. Cell Research. 16, 196 -202.

Turkson, J., Ryan, D., Kim, J.S., Zhang, Y., Chen, Z., Haura, E., Laudano, A., Sebti, S., \& Hamilton, A.D. (2001). Phosphotyrosyl peptides block stat3-mediated dna binding activity, gene regulation, and cell transformation. Journal of Biological Chemistry. 48, 45443-55.

Weaver, R.F. (2012). Molecular Biology. $5^{\text {th }}$ ed., McGraw Hill. Wei, D., Xiongdong, L.,Zheng, L., Wang, L., Frey, J.A., Gao, A.C., Peng, Z., Huang, S., Xiong, H.Q., Abbruzzese, J.L., \& Xie, K. (2003). STAT3 activation regulates the expression of vascular endothelial growth factor and human pancreatic cancer angiogenesis and metastasis. Oncogene. 22, 319-29.

Witte, S., \& Muljo, S.A. (2014). Integrating non-coding RNAs in JAK-STAT regulatory networks. JAKSTAT. 3(1): e28055.

Wormald, S., \& Hilton, D.J. (2004). Inhibitors of cytokine signal transduction targeting the interleukin-6/jak/stat pathway in human malignancies. Journal of Biological Chemistry. 279, 821-824.

Xiong, A., Yang, Z., Shen, Y., Zhou, J., \& Shen, Q. (2014). Transcription factor STAT3 as a novel molecular target for cancer prevention. Cancers (6)2, 926-57.

Yamaoka, K., Saharinen, P., Pesu, M., Holt, V.E.T., Silvennoinen, O., \& O'Shea, J.J. (2004). The Janus kinases (Jaks). Genome Biology. 12, 253.

Yang, C., Lee, H., Pal, S. Jove, V., Deng, J. Zhang, W., Hoon, D.S., Wakabayashi, M., Forman, S., \& Yu, H. (2010) B cells promote tumor progression via STAT3 regulatedangiogenesis. PLoS One. 8(5), e64159.

Zamzami, N., Brenner, C., Marzo, I., Susin, S.A., \& Koremer, G. (1998) Subcellular and submitochondrial mode of action of Bcl-2-like oncoproteins. Oncogene. 16, 2265-82. 